\title{
The acute effect of exercise intensity on vascular function in adolescents
}

Bert Bond $^{1}$, Siobhan Hind ${ }^{1}$, Craig A. Williams ${ }^{1}$ and Alan R. Barker ${ }^{1}$

${ }^{1}$ Children's Health and Exercise Research Centre, Sport and Health Science, College of Life and Environmental Sciences, University of Exeter, Exeter, EX1 2LU.

\section{Corresponding author:}

Dr Alan R. Barker

Children's Health and Exercise Research Centre

Sport and Health Sciences

College of Life and Environmental Sciences

University of Exeter

St Luke's Campus

Exeter

EX1 2LU

Tel: 44 (0)1392 722766

Fax: 44 (0)1392 724726

Email: A.R.Barker@exeter.ac.uk

Short title: Exercise intensity and vascular function in adolescents 


\section{ABSTRACT}

Introduction: Impairments in vascular function are present in asymptomatic youths with risk factors for cardiovascular disease. Exercise can promote vascular health in youth, but the effect of exercise intensity and the time course in response to acute exercise are unknown. Methods: Twenty adolescents (10 male, $14.1 \pm 0.3 \mathrm{y})$ on separate days, and in a counterbalanced order: 1) cycled at $90 \%$ of the gas exchange threshold (moderate-intensity exercise; MIE); 2) completed $8 \times 1$ min cycling at $90 \%$ peak power with 75 s recovery (high-intensity interval exercise; HIIE). The duration of MIE $(25.8 \pm 2.1 \mathrm{~min})$ was work-matched to HIIE (23.0 min). Macro- and micro-vascular function were assessed before, immediately post, and 1 and 2 hours after exercise by flow mediated dilation (FMD) and laser Doppler imaging (total reactive hyperaemia). Results: FMD was attenuated immediately after HIIE $(P<0.001$, $E S=1.20)$ but not MIE $(P=0.28, E S=0.26)$. Compared to pre-exercise, FMD was elevated 1 and 2 hours after HIIE $(P<0.001, E S=1.33$ and $P<0.001, E S=1.36)$ but unchanged in MIE $(P=0.67, E S=0.10$ and $P=0.72, E S=0.08)$. Changes in FMD were unrelated to shear or baseline arterial diameter. Compared to pre-exercise, total reactive hyperaemia was always greater after MIE $(P<0.02, E S>0.60$ for all $)$ and HIIE $(P<0.001, E S>1.18$ for all $)$. Total reactive hyperaemia was greater in HIIE compared to MIE immediately after $(P=0.03$, $E S=0.67)$ and 1 hour after $(P=0.01, E S=0.62)$ exercise, with a trend to be greater 2 hours after $(P=0.06, E S=0.45)$. Conclusion: Exercise intensity is positively associated with macro- and micro-vascular function 1 and 2 hours after exercise. Performing HIIE may provide superior vascular benefits than MIE in adolescents.

Key words: cardiovascular disease, endothelial function, youth, time course 


\section{INTRODUCTION}

2 Whilst the clinical manifestations of CVD are not detectable until adulthood, it is well established that the atherosclerotic process originates in the first decade of life (32). Impaired vascular function is thought to precede structural adaptations to the vessel wall (44), and both

5 macro- and micro-vascular function have been shown to be impaired in asymptomatic 6 adolescents with CVD risk factors $(8,19)$. Therefore, interventions which improve vascular 7 function in young people are warranted.

9 Data are available demonstrating that time spent performing vigorous-, but not moderate-, intensity physical activity is related to improved macrovascular function (17) and attenuated cardiometabolic risk (7) in youth. Additionally, exercise interventions have been shown to improve macrovascular function in obese adolescents (41). It has been suggested that changes in vascular function after a single exercise bout provide the foundation for these chronic adaptations $(3,12)$. Consequently, there is value in identifying the acute vascular responses to a single bout of exercise.

Previous studies with adults report conflicting results on the effects of acute exercise on macrovascular function, with some reporting increases $(16,18)$, decreases $(3,18)$ and no change (3) in flow mediated dilation (FMD). However, differences between exercise loads, modalities, the timing of the post exercise FMD measurement(s) (12) and the problems associated with reporting the ratio-scaled FMD statistic (1), currently limit our understanding of the FMD response to an acute bout of exercise. To our knowledge, only one study has assessed FMD immediately post exercise in young people (22). These authors reported that FMD immediately decreased after high-intensity, but not low-intensity, exergaming, and concluded that repeating high-intensity exergaming may provide a stimulus for favourable 
macrovascular adaptations. However, the exercise bouts were not work-matched in this study and FMD was only assessed immediately post exercise. Given that changes in vascular function within $\sim 2$ hours of exercise are thought to be biphasic in nature (12), it is important to document the time course of the change in vascular function after a single bout of exercise in youth to establish the influence of exercise intensity on the FMD response.

An impairment in microvascular reactive hyperaemia has been identified in asymptomatic children with clustered CVD risk (19) and it is thought that microvascular dysfunction may play a primary role in the pathogenesis of insulin resistance (25). Microvascular function has been shown to be elevated in adolescent football players compared to their untrained peers (29), however we are not aware of any study which has isolated the acute effect of exercise intensity on microvascular function in young people or adults. Furthermore, post exercise changes in microvascular reactive hyperaemia have been shown to be unrelated to FMD (31). Therefore, it is inappropriate to adopt post exercise changes in FMD as a surrogate of microvascular function.

The purpose of this investigation was to test the hypothesis that macrovascular function is immediately impaired, and then subsequently improved, following high-intensity interval exercise (HIIE), but remains stable following a work-matched bout of moderate-intensity exercise (MIE) in adolescents. A secondary aim was to identify the effect of exercise intensity on the time course of the microvascular response following exercise.

\section{METHODS}

Twenty 12 to 15 -year-old adolescents (10 males) volunteered to take part in this study. Written participant assent and parental consent were obtained before participation in the 
51 project, which was approved by the institutional ethics committee. Exclusion criteria included

52 the use of any medication or substance known to influence fat metabolism or vascular

53 function.

54

55 Experimental overview

56 This study required three visits to the laboratory and included a within-measures design. All exercise tests were completed using an electronically braked cycle ergometer (Lode Excalibur Sport, Groningen, the Netherlands).

Visit 1: Fitness assessment

61 Participants were habituated to the cycle ergometer before completing a combined ramp and supramaximal test to exhaustion to establish maximal oxygen uptake $\left(\dot{V} \mathrm{O}_{2} \max \right)$ (2). Pulmonary $\dot{V} \mathrm{O}_{2}$ was monitored throughout (Cortex Metalyzer III B, Leipzig, Germany) and the gas exchange threshold was identified as the disproportionate increase in carbon dioxide production $\left(\dot{V} \mathrm{CO}_{2}\right)$ relative to $\dot{V} \mathrm{O}_{2}$ and an increase in expired ventilation $(\dot{V} \mathrm{E}) / \dot{V} \mathrm{O}_{2}$ with no increase in $\dot{V} \mathrm{E} / \dot{V} \mathrm{CO}_{2}$. All exercise was performed on an electronically braked cycle ergometer

67 (Lode Excalibur Sport, Groningen, the Netherlands).

68

69

\section{Visits 2 and 3: Exercise interventions}

Participants completed two experimental conditions, separated by approximately one week. Following $\mathrm{a} \sim 12 \mathrm{~h}$ overnight fast, participants were transported to the laboratory at 08:00 and then consumed $30 \mathrm{~g}$ of commercially available Corn Flakes with $130 \mathrm{~mL}$ of skimmed milk. The macronutrient contribution of this breakfast is unlikely to have influenced endothelial function (40). 
At 08:45, participants rested in a darkened, temperature-controlled $\left(24^{\circ} \mathrm{C}\right)$ room for $15 \mathrm{~min}$ before the simultaneous assessment of macrovascular (flow mediated dilation (FMD)) and microvascular (laser Doppler perfusion imaging (LDI)) function (methods described below).

At 09:15, one hour after breakfast, participants completed on separate days and in a randomised order: 1) 30 min of continuous MIE at $90 \%$ of the gas exchange threshold; or 2) 23 min of HIIE (4). The HIIE bout consisted of a 3 min warm up at $20 \mathrm{~W}$, followed by $8 x$ 1 min intervals at $90 \%$ of the peak power determined from the ramp test to exhaustion, interspersed with $75 \mathrm{~s}$ of recovery at $20 \mathrm{~W}$, before a 2 min cool down at $20 \mathrm{~W}$. The duration of the MIE trial was calculated to match the total work performed during the HIIE bout. Participants provided a rating of perceived exertion (RPE) (43) in the final $10 \mathrm{~s}$ of exercise, before completing the 16-point Physical Activity Enjoyment Scale (PACES) (23) immediately after the exercise. After their final exercise trial, each participant was asked to identify which exercise bout they preferred.

Macro- and micro-vascular function were reassessed immediately after exercise cessation, with further measures 1 and 2 hours post exercise to facilitate comparison between extant literature in adults (12). Participants remained seated and were inactive at all times other than during the exercise bouts.

\section{Measures of vascular function}

FMD was measured using high resolution ultrasonography in duplex mode (Sequoia 512, Acuson, Siemens Corp, Aspen, USA) using a 12-14 MHz linear array transducer in accordance with recent guidelines (33) and our earlier work (4). Baseline and post occlusion brachial artery diameter was assessed during end diastole using validated ECG-gating 

diameter was measured for $1.5 \mathrm{~min}$. Endothelium-dependent vasodilation was calculated as the percentage increase in arterial diameter after a 5 min ischaemic stimulus induced by rapid forearm pneumatic cuff inflation (Hokanson, Bellevue, USA) to $220 \mathrm{mmHg}$ (33). The between-trial coefficient of variation for FMD was 9.7\%.

During the FMD protocol, microvascular function was simultaneously assessed using a laser point on the distal third of the forearm (11). High resolution data were collected at $4.33 \mathrm{~Hz}$, and then interpolated to $1 \mathrm{~s}$ averages before being smoothed using a $5 \mathrm{~s}$ moving average. Peak reactive hyperaemia (PRH) was defined as the highest point after occlusion. The total hyperaemic response was calculated in by determining the area under the post-occlusive reactive hyperaemic curve minus the baseline (pre-occlusion) blood flow (expressed as a percentage of $\mathrm{PRH}$ ), multiplied by the time taken for reactive hyperaemia to return to baseline (42). When calculated in this manner, the post-occlusive hyperaemic response is known to be nitric oxide independent (42), and accounts for differences in baseline skin perfusion. The between-trial coefficient of variation for PRH and the total hyperaemic response was 13.3 and $21.7 \%$ respectively.

\section{Standardisation of diet and physical activity}

120 With parental supervision, participants were asked to replicate their evening meal prior to each laboratory visit. Participants also completed a food diary during the 48 hour period immediately preceding each visit, which were subsequently assessed for total energy and macronutrient intake (CompEat Pro, Nutrition Systems, UK). Participants were instructed to avoid strenuous exercise and wear a tri-axial accelerometer on their wrist (GENEActiv, 
125 Activinsights Ltd, Cambridge, UK) during the 48 hour prior to each visit. Time spent performing moderate to vigorous activity was determined using established cut points for paediatric groups (13).

\section{Statistical analyses}

130 The primary outcome for macro-vascular function was the difference between logtransformed peak and baseline arterial diameter, adjusted allometrically for baseline diameter (1). Data were analysed using a linear mixed model with a random intercept (accounting for repeated measures within participants) plus fixed effects for condition (moderate/ high intensity), time (pre, post, 1-hour, 2-hour), and their interaction. As appropriate for a crossover trial, we also adjusted for any period effect. Differences on the log-scale were back-transformed to provide percent (ratio) effects. Point estimates are presented together with $95 \%$ confidence intervals. Additionally, the area under the curve for estimated shear rate was calculated from the last $30 \mathrm{~s}$ of occlusion until the time of peak dilation $\left(\mathrm{SR}_{\mathrm{AUC}}\right)(15)$, however FMD was not related to $\mathrm{SR}_{\mathrm{AUC}}$ at rest or at any point post exercise in either trial ( $P$ $140=0.21$ to $0.80, r=-0.1$ to 0.4$)$ which is consistent with other paediatric data $(4,34)$. Consequently, FMD was not normalised for $\mathrm{SR}_{\mathrm{AUC}}$.

Descriptive statistics were calculated using SPSS (version 19.0, Chicago, USA) and presented as mean \pm SD. Mean differences in descriptive statistics between boys and girls were analysed using independent samples $t$ tests. The mean differences in the physiological and perceptual responses of the boys and girls during HIIE and MIE were analysed using paired samples $t$ tests. Parameters of macro- and microvascular function were analysed using a mixed model ANOVA with trial (MIE, HIIE) and sex (male, female) as the main effects. 
parameters of macro- and micro-vascular function. Data were subsequently pooled for these

151

152

153

154 outcomes. Pairwise comparisons between means were interpreted using the $P$ value, 95\% confidence intervals and standardised effect sizes $(E S)$ to document the magnitude of the effect using the thresholds: small (0.2), moderate (0.5) and large (0.8) (9). Relationships between changes in vascular outcomes and mechanistically important variables were explored using Pearson's correlations.

\section{RESULTS}

Baseline participant characteristics are presented in Table 1. The maturation status for boys and girls was as follows; Tanner stage 2, $n=1$ and $n=0$; stage $3, n=3$ and $n=0$; stage 4, $n=5$ and $n=7$; stage $5, n=1$ and $n=3$. No differences in energy intake, individual macronutrient contributions, or time spent performing moderate to vigorous physical activity were apparent for boys or girls during the 48 hour preceding each laboratory visit $(P>0.50, E S<0.20$; Table 2).

The physiological and perceptual data from the exercise trials are presented in Table 3. All participants completed both exercise trials. The highest $\dot{V} \mathrm{O}_{2}$ achieved during the HIIE condition equated to $96 \pm 5 \%$. Average length of the MIE trial was $25.8 \pm 2.1 \mathrm{~min}$. Nine boys and eight girls indicated that they preferred the HIIE exercise bout.

\section{Macrovascular function}

Baseline arterial diameter, $\mathrm{SR}_{\mathrm{AUC}}$ and $\mathrm{FMD}$ are illustrated in Figure 1. A time by trial interaction was present for FMD $(P<0.001)$. No differences in mean FMD at baseline were apparent between trials $(P=0.62,95 \% \mathrm{CI}-1.2$ to $0.7, E S=0.12)$. Compared to baseline, FMD was attenuated immediately after HIIE $(P<0.001,95 \% \mathrm{CI}-4.4$ to $-2.3, E S=1.20)$, but was 
unchanged immediately following $\operatorname{MIE}(P=0.28,95 \%$ CI -1.5 to $0.4, E S=0.26)$.

Consequently, FMD was lower in HIIE compared to MIE immediately post exercise $(P<0.001,95 \% \mathrm{CI}-3.4$ to $-1.6, E S=1.57)$. FMD was not different to baseline 1 hour $(P=0.67$, $95 \% \mathrm{CI}-0.8$ to $1.2, E S=0.10)$ and 2 hours $(P=0.72,95 \% \mathrm{CI}-0.8$ to $1.1, E S=0.08)$ after MIE, however FMD was greater than baseline after HIIE at these time points $(P<0.001,95 \% \mathrm{CI}$ 1.7 to $3.7, E S=1.33$ and $P<0.001,95 \%$ CI 1.8 to $3.7, E S=1.36$, respectively). Consequently, FMD was greater in HIIE compared to MIE 1 hour $(P<0.001,95 \%$ CI 1.8 to $3.8, E S=1.31)$ and 2 hours $(P<0.001,95 \%$ CI 1.8 to $3.8, E S=1.33)$ post exercise. Changes in FMD post exercise were not related to age, maturity (Tanner stage) or aerobic fitness in either MIE or HIIE ( $r<0.43$ and $P>0.10$ for all).

There was a main effect of time $(P<0.001)$, but not trial $(P=0.28)$, or time by trial interaction $(P=0.75)$ for $\mathrm{SR}_{\mathrm{AUC}}$. Pairwise comparisons revealed that $\mathrm{SR}_{\mathrm{AUC}}$ was elevated immediately after exercise compared to baseline in MIE $(P<0.001,95 \%$ CI 206 to 564, ES=1.20) and HIIE $(P=0.001,95 \%$ CI 205 to $704, E S=1.31)$. There was also a trend for $\mathrm{SR}_{\mathrm{AUC}}$ to be greater 1 hour after MIE $(P=0.06,95 \% \mathrm{CI}-10$ to $358, E S=0.55)$ and $\mathrm{HIIE}(P=0.08,95 \% \mathrm{CI}-27$ to 394 , $E S=0.64$ ) compared to baseline. $\mathrm{SR}_{\mathrm{AUC}}$ was not different from baseline 2 hours after exercise for either trial $(P>0.14, E S<0.36$ for both).

There was a main effect of time $(P<0.001)$, but not trial $(P=0.68)$, or time by trial interaction $(P=0.09)$ for baseline arterial diameter. Baseline arterial diameter was greater immediately after exercise compared to pre exercise values in MIE $(P=0.03$, 95\% CI 0.01 to 0.22 , $E S=0.32)$ and HIIE $(P=0.01,95 \mathrm{CI} 0.05$ to $0.35, E S=0.51)$. Baseline diameter was not different from pre exercise values at any other point in either trial $(P>0.21, E S<0.20$ for all $)$. 


\section{Microvascular function}

201

202

203

204

205

206

207

208

209

210

211

212

213

214

215

216

217

218

219

220

221

222

223

Differences in parameters of microvascular function are presented in Figure 2. There was a main effect of trial $(P=0.002)$ and time $(P<0.001)$ for PRH, but no time by trial interaction $(P=0.14)$. There were no differences between trials in mean PRH at baseline $(P=0.51,95 \%$ CI -0.18 to $0.09, E S=0.12$ ). Compared to baseline, PRH increased immediately after MIE $(P=0.048,95 \%$ CI 0.02 to $0.46, E S=0.72)$ and HIIE $(P<0.001,95 \%$ CI 0.26 to 0.61 , $E S=1.16)$. PRH was greater in HIIE compared to MIE immediately after $(P=0.02,95 \% \mathrm{CI}$ 0.05 to $0.44, E S=0.73)$ and 1 hour after exercise $(P=0.002,95 \%$ CI 0.13 to $0.48, E S=0.67)$. There was also a trend for PRH to be greater in HIIE 2 hours after exercise $(P=0.08,95 \%$ CI -0.03 to $0.42, E S=0.43)$.

There was a main effect of trial $(P=0.01)$ and time $(P<0.001)$ for the total hyperaemic response, but no time by trial interaction $(P=0.17)$. There were no differences in total hyperaemic response between trials at baseline $(P=0.65,95 \% \mathrm{CI}-28$ to $18, E S=0.12)$. Compared to baseline, the total hyperaemic response was greater at all times after MIE $(P<0.02$ and $E S>0.60$ for all) and HIIE $(P<0.001$ and $E S>1.18$ for all). The total hyperaemic response was greater in HIIE compared to MIE immediately after $(P=0.03,95 \%$ CI 3 to 57 , $E S=0.67)$ and 1 hour after exercise $(P=0.01,95 \%$ CI 12 to $72, E S=0.62)$, with a strong trend for a statistical difference 2 hours after exercise $(P=0.06,95 \% \mathrm{CI}-1$ to $56, E S=0.45)$.

\section{DISCUSSION}

The purpose of this investigation was to establish the effect of exercise intensity on macroand micro-vascular function in adolescents, and to document the time course of the response. The novel findings from this study are: compared to baseline, 1) FMD is attenuated immediately following a single bout of HIIE but not MIE; 2) FMD is elevated 1 and 2 hours 
after HIIE, but unchanged in MIE; 3) PRH and total hyperaemic response are both increased during the 2 hours immediately following MIE and HIIE, and the magnitude of this increase is greater after HIIE than MIE. This is the first study to isolate the effect of exercise intensity and include serial measures of vascular function in adolescents after a single bout of exercise. The findings indicate that exercise intensity has an independent effect on macro- and microvascular function in young people, which likely have important implications for vascular health.

\section{Macrovascular function}

Our data demonstrate that an immediate post exercise nadir in FMD is present following HIIE but not MIE, which is consistent with work-matched data in adults $(3,18)$ and the only available data in young people (22). Mills et al. (22) hypothesised that this attenuation in FMD after high-intensity exercise might precede an increase in FMD, and might therefore be considered to be beneficial. However, these authors did not include serial measures of FMD in their investigation, and evidence of this response in endothelial function post exercise is scarce (18). Furthermore, the "high-intensity" exergaming trial included by Mills et al. elicited a mean $\dot{V} \mathrm{O}_{2}$ peak of $3.6 \pm 2.5$ metabolic equivalents, which the authors correctly classify as moderate-intensity (24). Therefore, the present study extends the work by Mills et $a l$ and, to our knowledge, is the first to confirm that the initial impairment in FMD following high-intensity exercise precedes an increase in macrovascular function, and that this improvement is present at least two hours later. Thus, exercise which elicits a greater acute challenge on the vasculature may be associated with larger increases in FMD in adolescents, and the evidence of a biphasic response in FMD post high-intensity exercise is compelling. 
Our failure to observe any changes in FMD immediately after MIE is consistent with the data provided by Mills et al. following "low-intensity" exergaming (22), however we extend their

251

252 findings and report that endothelial function remained unchanged during the 2 hours that followed. Interestingly, the lack of change in FMD in the hours after MIE is consistent with some $(3,18)$, but not all $(16,39)$ data in healthy adults. However, in addition to differences in exercise stimulus, timing of the FMD measurement and interpretation of the ratio-scaled FMD statistic $(1,12)$, an independent effect of training status (16) has been observed on the acute FMD response. Furthermore, evidence suggests that age might modulate vascular reactivity to the FMD protocol (34). Although we were unable to confirm a potential confounding effect of age, maturity (Tanner stage) or aerobic fitness on the change in FMD post MIE and HIIE, it appears that a direct comparison between our findings with apparently healthy adolescents and the available adult literature may be problematic.

Shear (when expressed as $\mathrm{SR}_{\mathrm{AUC}}$ ) is thought to be the main stimulus underlying the FMD response in healthy adults at rest (26). However, the relationship between $\mathrm{SR}_{\mathrm{AUC}}$ and FMD is not as robust following exercise (20). Indeed, we report here that FMD remained elevated in the hours following HIIE despite a steady decline in $\mathrm{SR}_{\mathrm{AUC}}$. The relationship between $\mathrm{SR}_{\mathrm{AUC}}$ and FMD has been shown to be weak in young people even at rest (34), a finding also observed in this study. It is therefore not surprising that differences in the FMD response 1 and 2 hours post exercise were independent of changes in $\mathrm{SR}_{\mathrm{AUC}}$. Considering that baseline arterial diameter remained unchanged 1 and 2 hours following MIE and HIIE, and that we followed recent statistical guidelines designed to partition out the influence of vessel calibre (1), our findings are also not explained by this factor. We are therefore unable to identify the mechanism(s) underlying the disparity in FMD response presented here. It has been speculated elsewhere that the initial impairment in FMD immediately following exercise 
relates to an increase in oxidative stress $(12,18)$, which would reduce the bioavailability of nitric oxide (6). Whilst we did not measure this outcome, an increase in oxidative stress following high-intensity exercise is not consistent with the augmented FMD response observed 1 and 2 hours after HIIE. Conversely, an exercise-intensity dependent increase in total antioxidant status has been reported during the hours following work-matched HIIE but not MIE (39), which would prevent the reduction in nitric oxide bioavailability associated with an increase in exercise-induced oxidative stress. However, this is not a consistent finding $(16,18)$, and we have previously reported that changes in FMD 1 hour after identical HIIE in adolescents were not related to total antioxidant status (4). Alternatively, given that the exercise bouts were work-matched in the present study, our data may be explained by a positive association between the intensity of exercise and subsequent activity of endothelial nitric oxide synthase. Indeed, data in adults demonstrate that brachial artery shear increases with the intensity of cycling exercise (35), and this has been demonstrated to play a leading role in the post exercise FMD response (36). We did not quantify brachial artery shear during the exercise bouts as this is technically challenging during HIIE. However, we have previously observed a reduction in postprandial systolic blood pressure in the 5 hours after HIIE, but not MIE, in adolescents (5), which would be consistent with an upregulation in endothelial nitric oxide synthase activity.

An interesting finding of the present study is that the magnitude of the increase in FMD observed 1 hour after HIIE was also present after 2 hours. Further study is needed to identify the precise decay in this favourable response after high-intensity exercise, although this benefit has been reported the following day in adults (39). Additionally, we have previously observed that a similar increase in FMD is present 4 hours after exercise despite the consumption of a meal which impaired FMD in a non-exercise control trial (4), whilst 
299 Sedgwick et al. reported an increase in postprandial FMD the day after repeated sprint cycling in adolescent boys (30). Therefore, a single bout of HIIE appears to provide a potent stimulus for macrovascular health, and may provide superior health benefits compared to MIE if repeated on a regular basis. Indeed, high-intensity interval training has been demonstrated to be more effectual in promoting macro-vascular function than moderateintensity training in adults at risk of vascular dysfunction (37), and offer superior improvements in FMD than a multi-disciplinary approach in overweight adolescents (38). Furthermore, only time spent performing vigorous-, but not moderate-, intensity exercise is related to vascular function in children (17).

\section{Microvascular function}

A novel feature of this investigation was the simultaneous assessment of post-occlusive reactive hyperaemia in the cutaneous circulation (11) during the FMD protocol. We have demonstrated that microvascular function is improved following both MIE and HIIE, and that the magnitude of this improvement is greater following HIIE. Furthermore, PRH and the total hyperaemic response to occlusion remained elevated 2 hours after exercise.

Our data show that transient improvements in microvascular function are possible following exercise without concomitant changes in FMD. No association has been demonstrated between FMD and reactive microvascular hyperaemia in adults post exercise (31), presumably because the post-occlusive cutaneous response is not mediated by nitric oxide (42). Our finding that micro-, but not macro-, vascular function was improved in the hours after MIE is probably testament to the different mechanisms underlying the post-occlusive hyperaemic response in our investigation, i.e. only the latter is NO-mediated (42).

323 Furthermore, the microvascular post-occlusive response may include both endothelial- 
independent and dependent pathways (11). It is therefore likely inappropriate to adopt measures of macrovascular health as an indication of global vascular function, especially as the earliest changes in vascular function due to the metabolic syndrome may be specifically linked to the capillary and arteriole beds, rather than the larger, conduit arteries (25). As a result, simultaneously assessing microvascular function alongside FMD may offer a novel insight regarding the effects of exercise intensity on vascular health.

We are the first to show that a single bout of MIE or HIIE can improve microvascular function in the hours following exercise, and that HIIE may provide a superior benefit. Whilst we were unable to identify the time course of the decay in these favourable responses post exercise, Gill et al. reported that endothelium-dependent microvascular function remained elevated 16-18 hours after 90 minutes of walking at $50 \% \dot{V} \mathrm{O}_{2} \max$ in adults (14). Therefore, repeating a single bout of exercise may have some utility in promoting microvascular function the following day, although this needs to be confirmed in adolescents. Conversely, there is evidence suggesting that the intensity of habitual physical activity may not influence microvascular endothelial function in adolescents (27). However, this study determined microvascular function by means that are considered to be NO-dependent, which is mechanistically disparate from our assessment (42). Currently, no study has identified the efficacy of HIIE training on microvascular health in asymptomatic adolescents. Further study is therefore needed to identify whether the acute benefits in microvascular function observed in the present study translate into meaningful benefits in this group with time.

\section{Considerations}

This is the first study to isolate the effect of exercise intensity on vascular function in adolescents. The strengths of this investigation include a work-matched design, control of 
prior physical activity and dietary factors, serial measures of macro- and micro-vascular function and allometric scaling of the FMD statistic. However, apart from reporting $\mathrm{SR}_{\mathrm{AUC}}$ and baseline arterial diameter, we are not able to provide any mechanistic data which could potentially explain the changes in vascular function following MIE and HIIE. A further limitation is that we were unable to measure the time course of these changes beyond 2 hours post exercise. Thus, the rate of decay in microvascular function following MIE and HIIE, and macrovascular function following HIIE remains unknown. We also cannot rule out that an increase in skin temperature following exercise influenced our measure of microvascular function. However, this unavoidable confounding effect is likely limited to the time point immediately post exercise as participants were acclimatised to the temperature-controlled $\left(24^{\circ} \mathrm{C}\right)$ room for all other vascular measures. Furthermore, our analysis of the post-occlusive reactive hyperaemic response accommodates differences in baseline perfusion (42). Finally, we are unable to comment on the interaction between exercise intensity and diurnal variation in FMD. Data in adults suggests that FMD could decline by $\sim 1 \%$ from baseline values over the course of our measurement period (28). However, the magnitude of this effect is far lower, and in the opposite direction, than the change observed following HIIE in the present study.

\section{CONCLUSION}

368 Our data indicate that the intensity of exercise has an independent effect on macro- and 369 micro-vascular function in adolescents. Specifically, macrovascular function was improved in the hours after HIIE but not MIE. Additionally, both exercise bouts promoted microvascular

371 function, although the magnitude of this increase was greater after HIIE. Therefore, it is likely that repeating high-intensity exercises may provide superior health benefits and lower 
cardiovascular disease risk than moderate-intensity activities. Given that HIIE was deemed to be more enjoyable than MIE, HIIE may provide an attractive, alternative to traditional MIE.

\section{ACKNOWLEDGEMENTS}

We thank Professor Alan Batterham for assistance with the allometric scaling of the flow mediated dilation data. We are also grateful to the staff and participants at Exmouth

Community College (Devon, UK) for their participation in this project.

\section{DISCLOSURES}

The authors have no conflicts of interest to disclose.

The results of the present study do not constitute endorsement by the ACSM.

\section{REFERENCES}

1. Atkinson G, Batterham AM. Allometric scaling of diameter change in the original flow-

2. Barker AR, Williams CA, Jones AM, Armstrong N. Establishing maximal oxygen uptake in young people during a ramp cycle test to exhaustion. Br J Sports Med. 2011;45(6):498-503.

3. Birk GK, Dawson EA, Batterham AM et al. Effects of exercise intensity on flow mediated dilation in healthy humans. Int J Sports Med. 2013;34(5):409-14.

4. Bond B, Gates PE, Jackman S, Corless L, Williams CA, Barker AR. Exercise intensity and the protection from postprandial vascular dysfunction in adolescents. Am J Physiol Heart Circ Physiol. 2015:ajpheart 000742015.

5. Bond B, Williams CA, Isic $\mathrm{C}$ et al. Exercise intensity and postprandial health outcomes in adolescents. Eur J Appl Physiol. 2015;115(5):927-36.

6. Cai H, Harrison DG. Endothelial dysfunction in cardiovascular diseases: the role of oxidant stress. Circ Res. 2000;87(10):840-4.

7. Carson V, Rinaldi RL, Torrance $B$ et al. Vigorous physical activity and longitudinal associations with cardiometabolic risk factors in youth. Int J Obes (Lond). 2014;38(1):16-21.

8. Celermajer DS, Sorensen KE, Gooch VM et al. Non-invasive detection of endothelial dysfunction in children and adults at risk of atherosclerosis. Lancet. 1992;340(8828):1111-5.

9. Cohen J. Statistical Power Analysis for the Behavioural Sciences. Hillsdale: Lawrence Erlbaum; 1988.

10. Corretti MC, Anderson TJ, Benjamin EJ et al. Guidelines for the ultrasound assessment of endothelial-dependent flow-mediated vasodilation of the brachial artery: a report of the International Brachial Artery Reactivity Task Force. J Am Coll Cardiol. 2002;39(2):257-65. 
11. Cracowski JL, Minson CT, Salvat-Melis M, Halliwill JR. Methodological issues in the assessment of skin microvascular endothelial function in humans. Trends in pharmacological sciences. 2006;27(9):503-8.

12. Dawson EA, Green DJ, Cable NT, Thijssen DH. Effects of acute exercise on flow-mediated dilatation in healthy humans. J Appl Physiol (1985). 2013;115(11):1589-98.

13. Evenson KR, Catellier DJ, Gill K, Ondrak KS, McMurray RG. Calibration of two objective measures of physical activity for children. J Sports Sci. 2008;26(14):1557-65.

14. Gill JM, Al-Mamari A, Ferrell WR et al. Effects of prior moderate exercise on postprandial metabolism and vascular function in lean and centrally obese men. J Am Coll Cardiol. 2004;44(12):2375-82.

15. Harris RA, Nishiyama SK, Wray DW, Richardson RS. Ultrasound assessment of flow-mediated dilation. Hypertension. 2010;55(5):1075-85.

16. Harris RA, Padilla J, Hanlon KP, Rink LD, Wallace JP. The flow-mediated dilation response to acute exercise in overweight active and inactive men. Obesity (Silver Spring). 2008;16(3):578-84.

17. Hopkins ND, Stratton G, Tinken TM et al. Seasonal reduction in physical activity and flowmediated dilation in children. Med Sci Sports Exerc. 2011;43(2):232-8.

18. Johnson BD, Padilla J, Wallace JP. The exercise dose affects oxidative stress and brachial artery flow-mediated dilation in trained men. Eur J Appl Physiol. 2012;112(1):33-42.

19. Khan F, Green FC, Forsyth JS, Greene SA, Morris AD, Belch JJ. Impaired microvascular function in normal children: effects of adiposity and poor glucose handling. J Physiol. 2003;551(Pt 2):705-11.

20. Llewellyn TL, Chaffin ME, Berg KE, Meendering JR. The relationship between shear rate and flow-mediated dilation is altered by acute exercise. Acta Physiol (Oxf). 2012;205(3):394-402.

21. Mancini GB, Yeoh E, Abbott D, Chan S. Validation of an automated method for assessing brachial artery endothelial dysfunction. Can J Cardiol. 2002;18(3):259-62.

22. Mills $A$, Rosenberg $M$, Stratton $G$ et al. The effect of exergaming on vascular function in children. J Pediatr. 2013;163(3):806-10.

23. Motl RW, Dishman RK, Saunders R, Dowda M, Felton G, Pate RR. Measuring enjoyment of physical activity in adolescent girls. Am J Prev Med. 2001;21(2):110-7.

24. Norton K, Norton L, Sadgrove D. Position statement on physical activity and exercise intensity terminology. Journal of science and medicine in sport / Sports Medicine Australia. 2010;13(5):496-502.

25. Pinkney JH, Stehouwer CD, Coppack SW, Yudkin JS. Endothelial dysfunction: cause of the insulin resistance syndrome. Diabetes. 1997;46 Suppl 2:S9-13.

26. Pyke KE, Tschakovsky ME. Peak vs. total reactive hyperemia: which determines the magnitude of flow-mediated dilation? J Appl Physiol. 2007;102(4):1510-9.

27. Radtke $T$, Kriemler $S$, Eser $P$, Saner $H$, Wilhelm M. Physical activity intensity and surrogate markers for cardiovascular health in adolescents. Eur J Appl Physiol. 2013;113(5):1213-22.

28. Ringqvist A, Caidahl K, Petersson AS, Wennmalm A. Diurnal variation of flow-mediated vasodilation in healthy premenopausal women. Am J Physiol Heart Circ Physiol. 2000;279(6):H2720-5.

29. Roche DM, Rowland TW, Garrard M, Marwood S, Unnithan VB. Skin microvascular reactivity in trained adolescents. Eur J Appl Physiol. 2010;108(6):1201-8.

30. Sedgwick MJ, Morris JG, Nevill ME, Barrett LA. Effect of repeated sprints on postprandial endothelial function and triacylglycerol concentrations in adolescent boys. J Sports Sci. 2014:1-11.

31. Shamim-Uzzaman QA, Pfenninger D, Kehrer $C$ et al. Altered cutaneous microvascular responses to reactive hyperaemia in coronary artery disease: a comparative study with conduit vessel responses. Clin Sci (Lond). 2002;103(3):267-73. 
32. Stary HC. Evolution and progression of atherosclerotic lesions in coronary arteries of children and young adults. Arteriosclerosis. 1989;9(1 Suppl):I19-32.

33. Thijssen DH, Black MA, Pyke KE et al. Assessment of flow-mediated dilation in humans: a methodological and physiological guideline. Am J Physiol Heart Circ Physiol. 2011;300(1):H212.

34. Thijssen DH, Bullens LM, van Bemmel MM et al. Does arterial shear explain the magnitude of flow-mediated dilation?: a comparison between young and older humans. Am J Physiol Heart Circ Physiol. 2009;296(1):H57-64.

35. Thijssen DH, Dawson EA, Black MA, Hopman MT, Cable NT, Green DJ. Brachial artery blood flow responses to different modalities of lower limb exercise. Med Sci Sports Exerc. 2009;41(5):1072-9.

36. Tinken $\mathrm{TM}$, Thijssen $\mathrm{DH}$, Hopkins $\mathrm{N}$ et al. Impact of shear rate modulation on vascular function in humans. Hypertension. 2009;54(2):278-85.

37. Tjonna $A E$, Lee $S J$, Rognmo $O$ et al. Aerobic interval training versus continuous moderate exercise as a treatment for the metabolic syndrome: a pilot study. Circulation. 2008;118(4):346-54.

38. Tjonna $\mathrm{AE}$, Stolen TO, Bye $\mathrm{A}$ et al. Aerobic interval training reduces cardiovascular risk factors more than a multitreatment approach in overweight adolescents. Clin Sci (Lond). 2009;116(4):317-26.

39. Tyldum GA, Schjerve IE, Tjonna AE et al. Endothelial dysfunction induced by post-prandial lipemia: complete protection afforded by high-intensity aerobic interval exercise. J Am Coll Cardiol. 2009;53(2):200-6.

40. Vogel RA, Corretti MC, Plotnick GD. Effect of a single high-fat meal on endothelial function in healthy subjects. Am J Cardiol. 1997;79(3):350-4.

41. Watts $\mathrm{K}$, Beye $\mathrm{P}$, Siafarikas $\mathrm{A}$ et al. Exercise training normalizes vascular dysfunction and improves central adiposity in obese adolescents. J Am Coll Cardiol. 2004;43(10):1823-7.

42. Wong BJ, Wilkins BW, Holowatz LA, Minson CT. Nitric oxide synthase inhibition does not alter the reactive hyperemic response in the cutaneous circulation. I Appl Physiol. 2003;95(2):504-10.

43. Yelling $M$, Lamb K, Swaine I. Validity of a Pictorial Perceived Exertion Scale for Effort Estimation and Effort Production During Stepping Exercise in Adolescent Children. European Physical Education Review. 2002;8(2):157-75.

44. Zeiher AM, Drexler $\mathrm{H}$, Wollschlager $\mathrm{H}$, Just $\mathrm{H}$. Modulation of coronary vasomotor tone in humans. Progressive endothelial dysfunction with different early stages of coronary atherosclerosis. Circulation. 1991;83(2):391-401.

(1)

(1)


Table 1: Participant characteristics

\begin{tabular}{lcccc}
\hline & Boys $(n=10)$ & Girls $(n=10)$ & $P$ value & $E S$ \\
\hline Age $(\mathrm{y})$ & $14.1 \pm 0.3$ & $14.1 \pm 0.3$ & 0.72 & 0.00 \\
Body mass $(\mathrm{kg})$ & $61.6 \pm 15.9$ & $54.9 \pm 4.6$ & 0.23 & 0.57 \\
Stature $(\mathrm{m})$ & $1.66 \pm 0.10$ & $1.65 \pm 0.08$ & 0.82 & 0.11 \\
$\dot{V} \mathrm{O}_{2 \max }\left(\mathrm{L} \cdot \mathrm{min}^{-1}\right)$ & $2.77 \pm 0.80$ & $2.04 \pm 0.36$ & 0.02 & 1.18 \\
$\dot{V} \mathrm{O}_{2 \max }\left(\mathrm{mL}^{-1} \min ^{-1} \cdot \mathrm{kg}^{-1}\right)$ & $44.8 \pm 6.4$ & $37.1 \pm 5.3$ & 0.01 & 1.26 \\
$\mathrm{GET}\left(\mathrm{L} \cdot \mathrm{min}^{-1}\right)$ & $1.36 \pm 0.35$ & $1.08 \pm 0.17$ & 0.04 & 1.02 \\
GET $\left(\% \dot{V} \mathrm{O}_{2 \text { max }}\right)$ & $49 \pm 4$ & $53 \pm 6$ & 0.11 & 0.78 \\
\hline
\end{tabular}

506

507

508

509

510

511

512

513

514

515

516

517

518

519

520

521

522

523

\section{9}

20

$\dot{V} \mathrm{O}_{2}$, oxygen uptake; GET, gas exchange threshold; $E S=$ effect size. Data presented as mean $\pm \mathrm{SD}$

Table 2: Accelerometer and food diary data during the 48 hours preceding each trial

\begin{tabular}{lcccc}
\hline & MIE & HIIE & $P$ value & $E S$ \\
\hline & & & & \\
Moderate-vigorous activity $\left(\mathrm{min} \mathrm{day}^{-1}\right)$ & $38 \pm 12$ & $36 \pm 15$ & 0.50 & 0.15 \\
${\text { Total energy intake }\left(\mathrm{kcal} \mathrm{day}^{-1}\right)}_{\text {Energy from carbohydrates }(\%)}^{1945 \pm 301}$ & $1887 \pm 341$ & 0.59 & 0.18 \\
Energy from fat $(\%)$ & $47 \pm 5$ & $47 \pm 5$ & 0.84 & $<0.01$ \\
Energy from protein $(\%)$ & $38 \pm 4$ & $38 \pm 6$ & 0.95 & $<0.01$ \\
& $15 \pm 4$ & $15 \pm 3$ & 0.73 & $<0.01$
\end{tabular}

MIE, moderate-intensity exercise trial; HIIE, high-intensity interval exercise trial $95 \% \mathrm{CI}=95 \%$ confidence limits for the true difference

Data have been pooled as ANOVA analysis revealed no main effect for sex 
524 Table 3: Physiological and perceptual responses to MIE and HIIE

\begin{tabular}{lcccc}
\hline & MIE & HIIE & $P$ value & ES \\
\hline Mean $\mathrm{HR}\left(\mathrm{b} \cdot \mathrm{min}^{-1}\right)^{*}$ & $129 \pm 14$ & $150 \pm 14$ & $<0.001$ & 1.50 \\
Mean $\mathrm{HR}\left(\% \mathrm{HR}_{\max }\right)^{*}$ & $66 \pm 6$ & $77 \pm 6$ & $<0.001$ & 1.83 \\
Mean $\dot{V} \mathrm{O}_{2}\left(\mathrm{~L} \cdot \mathrm{min}^{-1}\right)$ & $1.19 \pm 0.26$ & $1.49 \pm 0.37$ & $<0.001$ & 0.94 \\
Mean $\dot{V} \mathrm{O}_{2}\left(\% \dot{V} \mathrm{O}_{2 \text { max }}\right)$ & $51 \pm 8$ & $63 \pm 7$ & $<0.001$ & 1.60 \\
RER & $0.91 \pm 0.05$ & $1.03 \pm 0.06$ & $<0.001$ & 2.17 \\
RPE & $4 \pm 2$ & $7 \pm 1$ & $<0.001$ & 1.90 \\
PACES & $57 \pm 9$ & $65 \pm 7$ & $<0.001$ & 0.99 \\
Work performed $(\mathrm{kJ})$ & $117 \pm 18$ & $117 \pm 18$ & - & - \\
Energy Expenditure $(\mathrm{kJ})$ & $770 \pm 182$ & - & - & - \\
\hline
\end{tabular}

525

526

527

528

529

530

531

532

533

534

535

536

537

538

539

540

541

542

543

544

545

HR, heart rate; $\dot{V} \mathrm{O}_{2}$, oxygen uptake; MIE, moderate-intensity exercise trial; HIIE, high-

intensity exercise trial; $E S=$ effect size. Data presented as mean $\pm \mathrm{SD}$ and pooled for sex. $n=$ 20 apart from * where $n=18$ due to loss of telemetry 

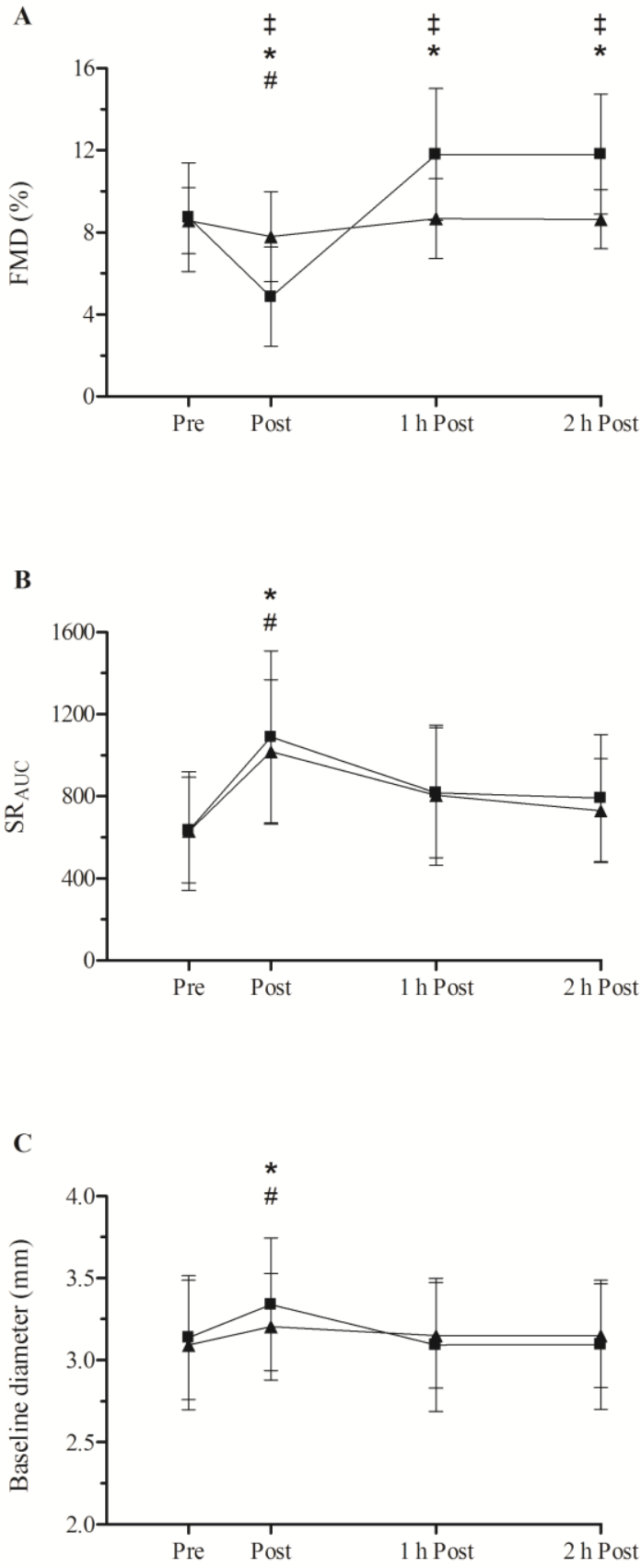

548 Figure 1 Mean differences in macro-vascular function pre and post moderate-intensity 549 exercise $(\boldsymbol{\Delta})$ and high-intensity interval exercise ( $\mathbf{})$. FMD, flow mediated dilation; SR $_{\mathrm{AUC}}$, 550 area under the curve for shear. Error bars represent the standard deviation. Significant 551 difference from pre exercise is denoted by ${ }^{\#}$ for moderate-intensity exercise and * for high552 intensity interval exercise. ${ }^{*}$ denotes significant difference between exercise trials. Refer to 553 text for specific $P$ values. 

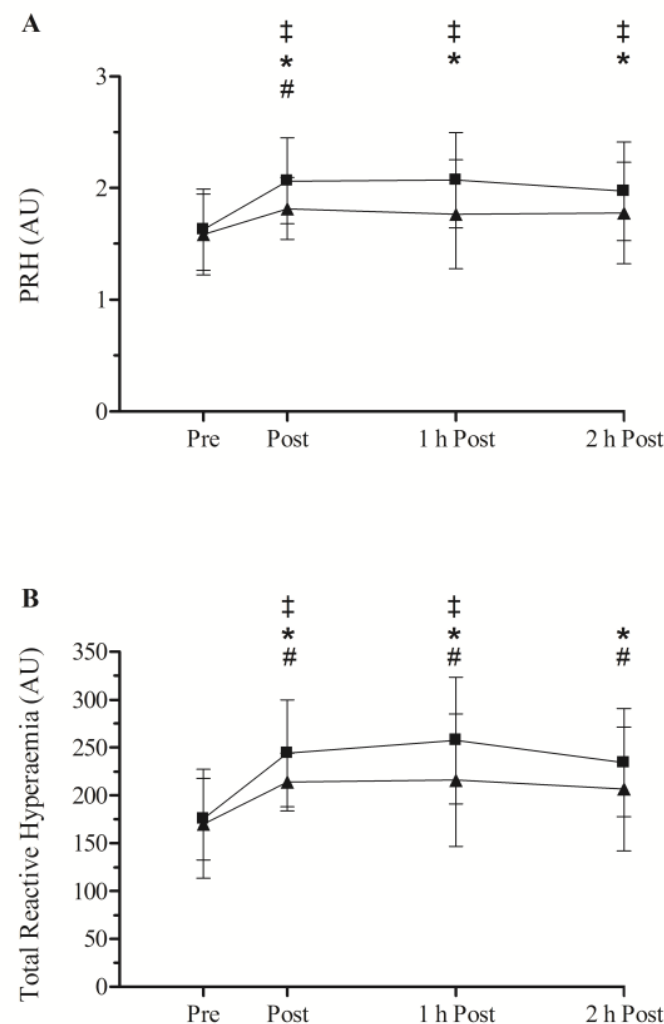

555

556 Figure 2 Mean differences in micro-vascular function pre and post moderate-intensity 557 exercise ( $\boldsymbol{\Delta}$ ) and high-intensity interval exercise (a). PRH, peak reactive hyperaemia; AU, 558 arbitrary units. Error bars represent the standard deviation. Significant difference from pre 559 exercise is denoted by ${ }^{\#}$ for moderate-intensity exercise and ${ }^{*}$ for high-intensity interval 560 exercise. ${ }^{*}$ denotes significant difference between exercise trials. Refer to text for specific $P$ 561 values. 